Revue québécoise de psychologie

Wuevécécolise

de psychologie

\title{
EXPRESSION DE LA SOUFFRANCE PSYCHIQUE CHEZ L'ADOLESCENT SCOLARISÉ EN SITUATION DE HANDICAP MOTEUR
}

Une analyse de l'impact de la qualité de l'environnement scolaire en contexte camerounais

EXPRESSION OF PSYCHIC SUFFERING OF SCHOOLED ADOLESCENT WITH PHYSICAL DISABILITY An analysis of impact of the quality of the school environment in Cameroon context

\section{Émilie Clarisse Tchokote}

Volume 40, numéro 3, 2019

LA SANTÉ MENTALE À L’ÉCOLE : ENJEUX ET DÉFIS DE LA PROMOTION ET L'ACCOMPAGNEMENT

MENTAL HEALTH AT SCHOOL: CHALLENGES AND CHALLENGES OF PROMOTION AND SUPPORT

URI : https://id.erudit.org/iderudit/1067546ar

DOI : https://doi.org/10.7202/1067546ar

Aller au sommaire du numéro

Éditeur(s)

Revue québécoise de psychologie

ISSN

2560-6530 (numérique)

Découvrir la revue

Citer cet article

Tchokote, É. C. (2019). EXPRESSION DE LA SOUFFRANCE PSYCHIQUE CHEZ L'ADOLESCENT SCOLARISÉ EN SITUATION DE HANDICAP MOTEUR : une analyse de l'impact de la qualité de l'environnement scolaire en contexte camerounais. Revue québécoise de psychologie, 40(3), 5-19.

https://doi.org/10.7202/1067546ar
Résumé de l'article

L'objectif de cet article est de saisir le sens de l'expression de la souffrance psychique chez l'adolescent en situation de handicap moteur inscrit dans un établissement secondaire inclusif dans un contexte d'imbroglio dans l'offre prônée par l'environnement scolaire en question. À travers la méthode clinique qualitative, l'utilisation de l'entretien clinique auprès de quatre cas d'adolescents inscrits au lycée de Biyem-Assi (Yaoundé-Cameroun), retrace l'expression de la souffrance psychique dans les discours des sujets (mentalisation par la tristesse, dépréciation de soi, angoisse, anxiété, développement d'un faux self, hyperadaptation). Sur le plan comportemental, on observe le retrait, le repli sur soi, la phobie face aux situations stressantes. 


\section{EXPRESSION DE LA SOUFFRANCE PSYCHIQUE CHEZ}

L'ADOLESCENT SCOLARISÉ EN SITUATION DE HANDICAP MOTEUR : une analyse de l'impact de la qualité de l'environnement scolaire en contexte camerounais

EXPRESSION OF PSYCHIC SUFFERING OF SCHOOLED ADOLESCENT WITH PHYSICAL DISABILITY: An analysis of impact of the quality of the school environment in Cameroon context

Émilie Clarisse Tchokote ${ }^{1}$

Université de Buea (Cameroun)

\section{INTRODUCTION}

La particularité connue de l'adolescence est le changement physiologique et morphologique qui survient chez le sujet et dont la transformation du corps prend une place centrale dans l'économie psychique de ce dernier. À cet âge développemental, les angoisses se focalisent sur le corps du fait des modifications et des remaniements dont il fait l'objet concernant notamment les aspects physiques, physiologiques, relationnels et psychologiques (Cloutier, 1996; Marcelli et Braconnier, 1988). Au-delà de la réalité biologique, le corps à l'adolescence, rend visible, non seulement les transformations liées à la puberté, mais aussi pose clairement le problème de l'identité chez le sujet. Ainsi, la particularité de cet âge développemental marque les mutations radicales que vit le sujet dans son mode de fonctionnement (Bee et Boyd, 2008).

Lorsque l'adolescent fait face à une situation de handicap notamment moteur, l'aspect somatique et l'aspect psychique de ce dernier seront une fois de plus atteints à travers la dimension corporelle et psychologique. De ce fait, un vécu douloureux sera observé du fait de la confrontation aux difficultés intenses et aux obstacles dans l'accomplissement des tâches liées à cet âge développemental.

Cette étude se focalise sur le vécu de l'adolescent en situation de handicap moteur scolarisé dans un contexte d'éducation inclusive au Cameroun. Dans ce sens, l'objectif de cet article est de saisir le sens de l'expression de la souffrance psychique chez l'adolescent en situation de handicap moteur inscrit dans un établissement secondaire inclusif.

Sachant que l'adolescence pose d'emblée la problématique de la place du corps, il va s'en dire, que l'adolescent qui est confronté à une situation de handicap physique pourra expérimenter une perception de

1. Adresse de correspondance : BP 7580 Yaoundé, Cameroun. Courriel : emilietchoko@yahoo.fr 
l'échec corporel. La blessure du corps vécu laisse des traces et occasionne un découragement du fait de la différence imposée et visible. Le bouleversement du corps dans un tel contexte peut induire une phobie de l'échec corporel, un mal-être et un malaise chez le sujet, car le corps vécu ne répond pas aux sollicitations de ce dernier.

De ce fait « le handicap constitue une épreuve, qu'il soit présent dès la naissance ou consécutif à un accident ou une maladie. Cette épreuve met en cause le rapport du sujet à son corps, au monde et à lui-même, et en fonction de son âge (nourrisson, enfant, adolescent ou adulte), aura des retentissements psychologiques différents. "(Bréjard et Pedinielli, 2014, p.59). La place du corps est donc au centre des préoccupations de l'adolescent puisque cela oriente et mobilise un regard à soi et un regard de l'autre (Joly, 2003) qui connote une représentation d'un corps " abimé ", " déformé " difficile à vivre. Le degré de difficulté est fonction de la nature du handicap moteur (congénital ou accidentel, mobilité réduite ou immobilité absolue avec trouble de déficit mental, sensoriel, sphinctérien, etc.) et dépend fortement du soutien accordé à l'adolescent dans une approche écosystémique. Comme l'avaient déjà suggéré Terrisse, Kalubi et Larivée (2007), le soutien accordé à la personne ayant des déficiences doit prendre en compte tous les sous-systèmes de l'écosystème, c'est-à-dire l'ontosystème (compétences personnelles, intrapsychiques), le microsystème (environnement familial, environnement scolaire), le mésosystème (prise en charge complète, aide psychologique et thérapeutique), le macrosystème (action de participation sociale, accomplissement des droits civiques, système éducatif et de santé) et l'exosystème (à travers les croyances et le regard que la société porte sur le handicap de l'individu).

L'étude s'intéresse au vécu de l'adolescent en situation de handicap moteur dans une approche microsystémique notamment l'environnement scolaire à caractère inclusif. Le vécu de l'élève en milieu scolaire cadre avec les approches de la qualité de vie à l'école (QDV). Bien que le concept de la qualité de vie à l'école manque de définition opérationnelle et universellement reconnue (Coudronnière et Mellier, 2016), il sert tout de même de repères conceptuels facilitant sa compréhension. C'est dans ce cadre que plusieurs dimensions tentent de définir les facteurs d'une qualité de vie à l'école, il s'agit des dimensions objectives, des dimensions structurales, des dimensions mixtes qui prennent en compte l'aspect objectif et l'aspect subjectif (Coudronnière et Mellier, 2016).

\section{PROBLÈME DE L'ÉTUDE ET CONTEXTE}

II est connu que la qualité de vie à l'école est un facteur qui favorise une intégration sociale harmonieuse et un bien-être des sujets à besoins 
spécifiques (lonescu, 1997). De ce fait, l'école est reconnue comme un microsystème capable de déterminer la santé mentale et assurer par ricochet un développement harmonieux chez l'adolescent. De ce fait, le niveau de satisfaction et le bien-être des élèves rendent compte de la qualité de vie à l'école (Bacro et Florin, 2013; Rask, Astedt-Kurki et Laippala, 2002).

Dans le cadre d'une éducation inclusive, l'adolescent en situation de handicap moteur scolarisé devra faire face non seulement aux exigences et aux offres de son milieu scolaire (microsystème), mais aussi bénéficier de l'adaptation du cadre à son contexte (handicap) (Plaisance et Schneider, 2013). II est connu que l'inclusion scolaire vise le développement des sentiments d'appartenance et d'acceptation des apprenants à besoins particuliers (Potvin et Lacroix, 2009; Thomazet, 2006). De ce fait, les représentations du bien-être à l'école varient selon les cultures et les politiques éducatives (Guimard, Bacro, Ferrière, Florin, Gaudonville et Thanh-Ngo, 2015).

L'étude s'intéresse au vécu des élèves en situation de handicap moteur dans un environnement scolaire censé favoriser les apprentissages et le bien-être à travers un système inclusif.

Or, le bien-être de l'adolescent scolarisé et en situation de handicap devrait être lié au plaisir d'aborder les situations éducatives et d'apprentissage, au bonheur et à la positivité des relations sociales, au développement positif des liens inter et intrapsychiques dans un contexte de rencontre et d'échange prôné par l'éducation inclusive. Dans le cadre de cette étude, le bien être de l'élève en situation de handicap est considéré dans son approche mixte à travers la dimension objective et subjective (Coudronnière et Mellier, 2016).

Le Cameroun s'est inscrit dans la mouvance de l'éducation inclusive telle que préconisée par la convention des Nations Unies (2006) relative aux droits des personnes handicapées, notamment en ce qui concerne l'enseignement primaire (qui est gratuit et obligatoire) ainsi que l'enseignement secondaire. C'est ce qui justifie que la loi $\mathrm{N}^{\circ} 2010 / 002 \mathrm{du}$ 13 avril 2010 portant protection et promotion des personnes handicapées au Cameroun, dans ses articles 30 et 31 (MINAS, 2010) stipule que les enfants et les adolescents ayant un handicap de quelque nature que ce soit, bénéficient de conditions d'éducation et d'apprentissage adaptées à leur état ainsi que des mesures particulières, dont la mise à disposition d'un matériel didactique adapté et de méthodes d'enseignement spécialisées. 
Dans le cadre de l'implémentation de ces différentes conventions et lois, deux lettres circulaires conjointes des ministres des Enseignements secondaires et des Affaires sociales ont vu le jour. II s'agit de la lettre circulaire $N^{\circ} 34 / 06 / L C / M I N E S E C / M I N A S$ du 02 août 2006 relative à l'admission des enfants en situation de handicap et ceux nés de parents handicapés indigents dans les établissements publics d'enseignement secondaire; de la lettre circulaire $N^{\circ} 283 / 07 / L C / M I N E S E C / M I N A S$ du 14 août 2007 relative à l'identification des enfants handicapés et ceux nés des parents handicapés indigents inscrits dans les établissements publics d'enseignement secondaire et à leur participation aux examens officiels.

Ces lettres circulaires permettent non seulement de lutter contre la sous-scolarisation des enfants en situation de handicap, mais aussi de faciliter l'accès à une scolarisation aux enfants nés de parents handicapés indigents. Aussi, la situation d'inclusion scolaire prônée dans les textes et conventions en vigueur vise le développement harmonieux de cette catégorie d'élèves et le sentiment d'appartenance et d'acceptation susceptible d'accroître le sentiment de compétence et la réussite éducative.

Dès lors, comment est-ce que la qualité de l'environnement scolaire à caractère inclusif contribue à la mise en œuvre du bien-être chez l'adolescent en situation de handicap? Dans un contexte d'imbroglio dans les types de médiations offertes au sujet (médiation éducative, pédagogique, liée à l'accessibilité et à la résolution des conflits, etc.), il est question de laisser s'exprimer le vécu de l'adolescent scolarisé en situation de handicap de manière objective et subjective.

L'hypothèse est que la qualité de l'environnement scolaire inclusif, influence le vécu de l'adolescent en situation de handicap moteur et occasionne un retentissement psychologique s'exprimant aisément par une souffrance psychique chez ce dernier.

\section{MÉTHODE}

Participants et site de l'étude

L'étude s'inscrit dans la logique compréhensive et cherche à cerner l'impact de la qualité de l'environnement scolaire sur la souffrance psychique exprimée par l'adolescent en situation de handicap moteur sans autre trouble associé. C'est une étude de quatre cas d'adolescents âgés de 14 à 16 ans en situation de handicap moteur régulièrement inscrits au lycée de Biyem-Assi situé dans la ville de Yaoundé, région du centre du Cameroun. Le lycée de Biyem-Assi est choisi parce qu'il fait partie des établissements d'éducation inclusive au Cameroun. 
Outil de collecte des données et procédures

La méthode clinique de recherche a été utilisée afin de permettre une saisie du sujet concret en situation actuelle et en rapport à son histoire personnelle et à son contexte (Douville, 2006; Muchielli, 2009). La collecte des données s'est faite à base d'un guide d'entretien thématique permettant de retracer les modalités de la qualité de l'environnement scolaire ainsi que son influence sur le bien-être psychologique du sujet. Le choix de cet outil repose sur la possibilité à faciliter le recueil d'une masse importante d'informations utiles dans la compréhension du vécu de l'élève en situation de handicap dans un contexte scolaire à caractère inclusif. Aussi, cette posture donne aux participants la possibilité de s'exprimer de manière libre et sans contrainte.

Les entretiens ont eu lieu dans la salle de service des conseillers d'orientation du lycée de Biyem-Assi. Chaque entretien durait en moyenne une heure et se faisait le mercredi après-midi étant donné que c'est une demi-journée de cours. Deux entretiens ont été réalisés par cas (huit entretiens au total). Chaque adolescent était informé du but de l'étude (contribuer à l'étude de l'analyse de la qualité de vie et de l'environnement scolaire), cependant, nous ne leur donnions pas notre hypothèse afin d'éviter tout biais dans leurs verbalisations.

À travers la garantie de la confidentialité de la participation des sujets à l'étude, les pseudonymes ont été attribués aux sujets de l'étude ainsi que les fiches de consentement éclairé afin de respecter la volonté des participants à participer ou pas à l'étude. Cette procédure nous permettait ainsi de nous conformer à l'un des grands principes de l'éthique de la recherche. Les entretiens étaient enregistrés grâce au dictaphone et son utilisation était signalée à l'avance aux adolescents.

\section{Technique d'analyse}

Les données collectées ont été analysées à travers la technique d'analyse thématique de contenu avec ancrage sur les unités de sens contenues dans les discours des participants pouvant permettre un fondement empirique de l'analyse.

\section{RÉSULTATS}

II est question de présenter les participants de l'étude à travers les données idiographiques des sujets (Fati, Reine, César, Antoine), les conditions de la survenue du handicap ainsi que les données liées à leur scolarisation. 


\section{PRÉSENTATION DES PARTICIPANTS}

\section{Fati}

De sexe féminin, Fati est élève en classe de troisième allemand au lycée de Biyem-Assi. Âgée de 15 ans, elle est issue d'une famille monogamique et occupe le troisième rang d'une fratrie de quatre. Fati vit actuellement avec sa sœur aînée âgée de 24 ans et qui joue le rôle de tutrice. Son père décède lorsqu'elle a quatre ans et sa mère se remarie pour aller vivre dans la région du littoral avec son époux. Au moment de notre rencontre, nous observons qu'elle est atteinte d'un handicap moteur des deux jambes et connaît des difficultés énormes pour se déplacer malgré l'utilisation de ses deux béquilles. Elle révèle que son handicap fait suite à une constatation de l'atrophie de ses muscles des membres inférieurs alors qu'elle est âgée de deux ans. D'allure très timide et moins expressive, elle révèle que sa scolarisation a été très perturbée du fait de son handicap et du manque de moyens financiers; néanmoins, elle débute sa scolarisation à l'âge de six ans au $\mathrm{CNRPH}^{2}$. Elle fréquente le lycée de Biyem-Assi depuis la classe de cinquième.

\section{Reine}

De sexe féminin, Reine est élève en classe de première A4 allemand au lycée de Biyem-Assi. Âgée de 16 ans, elle est issue d'une famille monogamique et est l'aînée d'une fratrie de quatre. Elle vit actuellement avec sa grand-mère à Yaoundé (capitale politique du Cameroun) et ses parents vivent à Douala (à $235 \mathrm{~km}$ de Yaoundé) avec le reste de la fratrie. À l'observation, Reine est atteinte d'un handicap moteur d'une jambe et se déplace sans béquille, mais très péniblement. Elle révèle que son handicap a été acquis à l'âge de six ans suite à une maladie grave qui a occasionné une déformation de ses membres inférieurs. Malgré plusieurs interventions chirurgicales qui se sont soldées par des échecs, Reine nous confie qu'elle est contrainte à vivre avec «le fer dans son pied» et selon sa plus récente consultation, son médecin lui a révélé une complication qui pourrait se solder par une amputation de sa jambe droite. Selon ses verbalisations, elle débute sa scolarisation timidement à l'âge de six ans et fréquente le lycée de Biyem-Assi depuis la classe de sixième.

\section{César}

De sexe masculin, César est élève en classe de Première D2 au lycée de Biyem-Assi. Âgé de 16 ans, il est issu d'une famille monogamique et est le benjamin d'une fratrie de sept. Orphelin de père, il vit actuellement

2. CNRPH- Centre national de réhabilitation des personnes handicapées- Cardinal Paul Émile Léger, située à Etoug-ébé à Yaoundé -Cameroun. 
chez son grand frère tandis que sa mère vit à Dschang (dans la région de l'Ouest du Cameroun) (à 341,8 km de Yaoundé).

Au moment de sa rencontre, nous observons qu'il est en situation de handicap moteur d'une jambe dû à un déboîtement du bassin depuis l'âge de huit ans. Malgré les multiples recours aux traitements traditionnels, César se déplace sans béquille et affirme être toujours dans des difficultés énormes lorsqu'il est en mouvement.

\section{Antoine}

De sexe masculin, Antoine est élève en classe de seconde A4 au lycée de Biyem-Assi. Âgé de 16 ans, il est issu d'une famille monogamique et occupe le deuxième rang d'une fratrie de cinq. II vit actuellement avec son oncle maternel, ses parents vivent à Bafoussam (dans la région de l'Ouest du Cameroun) située à 296,8 km de Yaoundé. À l'observation, Antoine présente un handicap moteur d'une jambe et se déplace à l'aide d'une béquille. D'allure peu expressive, il révèle que son handicap fait suite à un accident sur la voie publique lorsqu'il a été heurté par une motocyclette.

\section{QUALITÉ DE L'ENVIRONNEMENT SCOLAIRE EN CONTEXTE D'INCLUSION}

La particularité d'un établissement scolaire à caractère inclusif est d'offrir un cadre et des dispositifs scolaires facilitant l'adaptation de tous les apprenants, quelles que soient leurs différences. Dans ce contexte, les aménagements infrastructurels et organisationnels sont nécessaires pour réduire les difficultés ressenties par l'élève en situation de handicap. Dans ce sens, les questions liées à l'accessibilité, à l'adaptabilité, au soutien et à l'accompagnement trouvent toutes leurs significations pour pouvoir induire chez le sujet un sentiment de bien-être. Concernant l'offre et l'accessibilité des dispositifs scolaires, il s'agira de pouvoir accéder à sa salle de classe, aux aires de jeux, aux toilettes, à la cantine scolaire, à la hauteur du tableau et aux autres lieux de service nécessaires tels que le bloc administratif, la surveillance, l'infirmerie, le service de conseillers d'orientation, etc.

Les sujets de l'étude ont laissé transparaître un mal-être lors des entretiens et selon leurs verbalisations, ils ont fait état de la difficulté d'accès à leur bâtiment, aux salles de cours, aux toilettes, à l'infirmerie et à la cantine scolaire lors des heures de pause. Le sujet Antoine mentionne que :

Ma salle de classe est à l'étage et à chaque fois, je dois grimper pour y arriver. Mais c'est vrai, il m'arrive parfois de ressentir de la douleur au niveau des hanches et des cuisses [...] Même après avoir grimpé les 
escaliers, j'ai toujours mal![...] Aux heures de pause, je ne peux pas aller comme mes camarades à la cantine par exemple, non! (bégaie) je ne peux pas, parce qu'il faut parcourir une longue distance et après, je me sens très fatigué!

La situation décrite par Antoine démontre à suffisance que la qualité du dispositif infrastructurel rappelle sans cesse l'incapacité du sujet à s'adapter à son environnement scolaire du fait de sa déficience. Cette difficulté est ressentie très péniblement chez les autres sujets de l'étude (Reine, César et Fati). Reine stipule que "j'ai été vraiment traumatisée, ma classe m'a marquée parce que comme j'avais le plâtre et les chaussures en fer, je n'arrivais pas à grimper facilement parce que la classe était à l'étage, c'est très difficile! ».

Face à ces difficultés, les sujets de l'étude éprouvent des malaises et sont contraints à la dépendance forcée vis-à-vis de leur camarade, ce qui renforce leur sentiment de mal-être. C'est le cas de Fati qui n'hésite pas à bénéficier de l'aide de sa camarade dans une sorte de dépendance absolue pour pouvoir se mettre à l'aise. Elle précise que :

Pour aller aux toilettes, c'est vraiment difficile, généralement, je me fais accompagner par ma camarade qui est ma seule amie. Même si le professeur est là, il nous autorise à sortir à deux pour qu'elle me facilite la tâche aux toilettes [...] Je suis très mal à l'aise et vraiment gênée, surtout que je suis obligée de me courber pour faire mes besoins, il n'y a pas de pot de toilette, c'est un espace aménagé qui est très bas et ne me permet pas de me mettre à l'aise convenablement. Je suis vraiment peinée et à chaque fois, ça me salit et j'ai honte! À l'heure de pause, si je veux quelque chose, j'envoie mon amie me faire la commission, parce que c'est trop difficile pour moi d'arriver là-bas!

Le malaise est perceptible dans ce discours et témoigne d'une qualité de vie à l'école qui ne favorise pas le plein épanouissement et le bien-être du sujet.

\section{OFFRE PÉDAGOGIQUE ET FORME DE MÉDIATIONS EN SITUATION INCLUSIVE}

Dans une situation d'éducation inclusive, la qualité de la médiation aussi bien éducative que pédagogique doit pouvoir participer au bien-être psychique du sujet-apprenant. L'intervenant ou le professionnel qui agit dans le but de faciliter l'apprentissage chez le sujet à travers la médiation pédagogique (Cardinet, 1998; Lenoir, 2009), doit mettre en œuvre des stratégies comportementales, cognitives et des actions précises afin de susciter la résolution des situations problèmes liées à l'apprentissage chez le sujet (Myara, 2017). Dans leurs verbalisations, les sujets de l'étude (Fati, Reine, César et Antoine) expriment la difficulté majeure à se faire 
accompagner pédagogiquement dans l'acte d'apprendre. Cette difficulté est liée en grande majorité à la perception du vécu du handicap chez l'apprenant lui-même d'une part, et à l'absence d'implication réelle des différents intervenants dans la médiation éducative d'autre part. Dans ce sens, le sujet Fati précise que :

Pendant les séances d'apprentissage, je n'aime pas prendre la parole et les enseignants ne me donnent pas la parole...! Je n'aime pas du tout m'exprimer devant les gens, j'ai peur et je n'ai pas envie qu'on me regarde, ça me fait trop honte! Même si je ne comprends pas le cours, je préfère me taire et rester seule!

Ce discours laisse transparaître le signe d'un malaise profond du sujet en lien avec la perception de son propre corps (corps déformé, étrange, inquiétant) qui rappelle l'inquiétante étrangeté et suscite l'effroi chez celui qui le regarde. Pour Korff-Sausse (1996), la situation de déficience motrice traduirait une figure de l'inquiétante étrangeté, car, elle serait un miroir tendu où se reflètent des puissances enfouies chez tout individu, d'où la confession faite par Fati « j'ai peur et je n'ai pas envie qu'on me regarde, ça me fait trop honte! ». Le sujet est ici submergé par la gêne vis-à-vis de ses camarades et du fait de la difficulté vécue lors de la mise à l'épreuve des situations pédagogiques, entraînant une interaction enseignant- élève.

La conséquence est vite perçue au niveau de l'effort de compréhension et d'apprentissage du sujet avec un rendement peu probant. Or, comme l'avait déjà suggéré Tchokote (2019), la situation inclusive devrait amener les intervenants (en occurrence les enseignants) à aider l'apprenant à s'intéresser à la situation pédagogique tout en mettant en œuvre des stratégies efficaces de soutien à l'action de résolution des conflits potentiels ou réels d'apprentissage.

Le sujet César, en évoquant ses difficultés lors de l'évaluation des activités physiques et sportives, précise que :

J'avais fait l'EPS (Education Physique et Sportive) par écrit et j'ai eu $0,5 / 20$ sur mon relevé de notes. Ça gêne ma scolarité. Le handicap fait en sorte que je me retrouve toujours en train de reprendre les classes parce que je ne suis pas à l'aise. Les stratégies d'évaluation en EPS par exemple, ne tiennent pas comptent de ma situation et ça me fait regretter et je me dis que si je n'étais pas handicapé, j'aurais aussi fait la pratique comme les autres et j'aurais réussi! Ceux qui sont aptes ont l'avantage parce qu'ils font la pratique, alors que nous qui sommes handicapés, on nous impose l'écrit, pourtant, on n'enseigne pas ça en classe! Ça nous désavantage, je suis parfois obligé d'aller voir l'enseignant pour expliquer ma situation!

À travers ce récit, on comprend aisément que l'élève est gêné de ne pas être compris et est pénalisé pour sa situation de handicap, car il est 
évalué effectivement là où réside sa limitation et sa faiblesse. Cette situation renforce le désavantage chez le sujet et accentue le malaise ressenti par ce dernier. Cette gêne est exacerbée par le manque de suivi de la part de l'enseignant d'éducation physique et sportive qui n'a pas été formé à l'accompagnement des élèves à besoin particulier. Néanmoins, certains intervenants font montre d'un souci d'accompagnement à l'action éducative; ce qui reste encore insuffisant lorsqu'il est question du soutien à la personne en situation de handicap. Cette situation pourrait s'expliquer par le manque de formation adéquate dans le processus d'accompagnement de cette catégorie d'élève en situation d'éducation inclusive au Cameroun.

Dans cette logique, le sujet Reine précise que :

Les enseignants enseignent bien, mais je n'aime pas qu'on m'interroge, je n'aime pas être vue, je n'aime pas qu'on s'intéresse à moi. Mais, il y a certains enseignants qui me demandent de partir au tableau, parfois je le fais, mais parfois, je ne pars pas [...]. Et lorsque le professeur parle et explique, même si j'ai une question, je ne lève pas le doigt pour m'exprimer! Je laisse et j'attends de voir si quelqu'un d'autre peut poser la question. Je préfère prendre un camarade qui peut m'expliquer un cours, mais pas aller directement voir l'enseignant.

On constate ici que les représentations liées au handicap occupent une place centrale dans l'économie psychique du sujet avec un ancrage sur le corps du sujet " corps difforme, corps cassé, corps abîmé » qui évite le regard de l'autre «[...] je n'aime pas être vu, je n'aime pas qu'on s'intéresse à moi [...] ». Dans ce contexte, l'impact est cruellement visible dans le rapport pédagogique avec un manque de volonté manifeste dans la participation aux activités cognitives et aux interactions impliquant l'élève en situation de handicap et d'autres acteurs (élèves, enseignants).

\section{EXPRESSION DE LA SOUFFRANCE PSYCHIQUE ET TRAVAIL DE MENTALISATION CHEZ L'ADOLESCENT SCOLARISÉ EN SITUATION DE HANDICAP MOTEUR}

L'expression de la souffrance psychique est retracée dans les récits des sujets qui témoignent d'un vécu douloureux et d'un malaise psychologique dans un tel contexte. Ce malaise est d'abord vécu sur le plan physique (difficulté à se mouvoir et à agir sur son environnement) du fait de l'absence ou de l'insuffisance dans la mise en œuvre du cadre scolaire adéquat à la condition du sujet en situation de handicap en général et du handicap moteur en particulier ainsi que des dispositifs inappropriés. (Reine, César, Antoine et Fati). Ce malaise est ensuite vécu sur le plan pédagogique du fait du manque d'implication de certains élèves en situation de handicap par les enseignants (Fati, César et Antoine) et du 
poids des représentations du handicap couplé de l'impact du regard de soi sur soi et du regard de l'autre sur soi (Reine, Fati et César).

Concernant l'impact du regard de l'autre et du stigmate, Antoine évoque en ces termes le malaise ressenti lorsque ses camarades se moquent de lui :

Tout le monde ne peut pas m'apprécier, certains camarades viennent quand même vers moi et ça me réconforte. Mais il y a ceux qui m'envoient certains mots comme 'eboa' (en référence à un célèbre artiste camerounais handicapé moteur de regretté mémoire), et je me sens mal et triste quand ils disent ça! il y a même d'autres camarades qui vont même loin et ils imitent ma façon de marcher, mes mouvements, et se moquent! et beaucoup d'autres se mettent à rire! c'est vrai que ça me blesse énormément, mais j'essaye de garder mon calme! face à ces choses, je gère ça comme ça, comme je peux sans aller voir le surveillant et je considère qu'ils sont ignorants, ils ne savent pas ce qu'ils font et quand c'est comme ça, j'essaye un peu de rester dans mon coin!

De ce récit, il ressort que le sujet qui fait face à une incompréhension ou qui est victime de stigmatisation se sent obligé d'adopter l'isolement et le retrait pour éviter une certaine confrontation avec les autres apprenants. De ce fait, le sentiment qui s'en suit est celui de la mise à l'écart pour certains (Antoine, Fati, César), de l'évitement pour d'autres (Fati, Reine, César), car le cadre de l'institution ne semble pas s'adapter au sujet en situation de handicap moteur. Dans un tel contexte, le sujet vit de manière subjective et objective une souffrance pouvant altérer son processus d'apprentissage. L'expression de cette souffrance est perçue à travers les mots et sous un mode mentalisé à l'instar de la tristesse, de l'angoisse qui est quasi permanente, de l'anxiété et de la dépréciation de soi. C'est le cas de Fati qui, évoquant les difficultés liées à l'accessibilité et au handicap, précise : "Ça ne me plaît pas de vivre comme ça! Je ne me sens pas comme les autres, ça me fait très mal d'être différente des autres et de ne pas pouvoir faire comme les autres ici au lycée! ça me fait vraiment mal! ».

La tristesse mêlée au chagrin et à la colère est vécue par le sujet Reine qui parvient à formuler dans son discours les raisons de ce ressenti lorsqu'elle précise que « je me sens mal à l'aise et triste ici à l'école, parce que moi-même d'abord, je ne m'accepte pas du fait que ...(bégaie) c'est resté comme ça! (le pied) et c'est aussi dû à la colère et au chagrin! J'aurais préféré qu'on m'ampute le pied! (hausse le ton) ».

On constate que la phobie ressentie face au regard des autres (camarades) vient amplifier le vécu pénible et douloureux des sujets qui l'expriment d'ailleurs aisément. Reine dit à propos que "ce qui me dérange beaucoup c'est l'avis des gens, le regard des autres ». Antoine et César manifestent le même ressenti et éprouvent des difficultés lorsqu'ils 
doivent faire des efforts supplémentaires pour s'adapter au contexte scolaire. César précise que "lorsque je pense aux efforts que je dois faire en plus, ça me fait très mal! ». Aussi, le manque d'implication dans l'acte d'apprendre est signalé dans les discours des sujets avec pour conséquence un malaise psychologique, un repli sur soi, une phobie face aux situations d'apprentissage et une hyperadaptation (Reine, Fati et César). C'est dans ce sens que César souligne que "je suis assis au premier banc et il y a les enseignants qui se fichent de savoir que nous sommes là et ça me dérange! II faudrait qu'ils soient plus attentifs et regardants vis-à-vis de nous! J'accepte la situation là comme ça, parce que je n'ai pas le choix. Tout ça gêne énormément ma scolarité! »

Face à la souffrance vécue et exprimée, les sujets mettent en œuvre des stratégies adaptatives pour amoindrir le ressenti pénible de la situation du handicap. C'est le cas de Reine qui n'hésite pas à faire de la comparaison lorsqu'elle dit :

\begin{abstract}
Je me dis qu'il n'y a personne qui soit parfaite, il y a même les gens qui sont plus que moi (handicapé). Moi j'ai même la grâce que je peux marcher sur mes jambes. II y a certaines personnes que je vois à l'hôpital qui sont pires que moi et plus handicapé que moi. J'essayais de me mettre à leur place et ça me permet de m'accepter comme je suis! Depuis que je suis en première et il y a les gens qui m'ont déjà connue comme ça, donc il m'accepte comme ça. Mais de temps en temps, le mal revient et c'est toujours en moi!
\end{abstract}

On constate que la souffrance refait surface malgré toutes les stratégies mises en œuvre par les sujets de l'étude pour éviter un tel ressentiment, " le mal revient et c'est toujours en moi! » (Reine).

La persistance de la souffrance chez l'élève en situation de handicap moteur dans son environnement scolaire, amène à s'intéresser et à discuter de la qualité de l'environnement, de la qualité des liens établis entre les différents intervenants dans un tel contexte, ainsi que de la place et du rôle des acteurs de ce système.

\title{
DISCUSSION ET CONCLUSION
}

Vivre avec le handicap à l'adolescence a toujours été considéré comme un périlleux passage (Bon, 2007). Dès lors qu'il est question pour l'adolescent en situation de handicap de vivre dans un contexte scolaire qui promeut l'éducation inclusive, on va s'intéresser au vécu de ce dernier dans son environnement scolaire qui offre la même éducation à tous les élèves qui s'y retrouvent. II est connu que le degré de satisfaction des élèves est corrélé à la qualité de vie à l'école et l'environnement scolaire peut offrir un espace de reconstruction aux élèves qui vivent des situations aversives à travers un processus de résilience (Anaut, 2006). Peut-on dire de même des élèves en situation de handicap qui vivent dans un 
environnement scolaire inclusif, mais qui ne semblent pas bénéficier des atouts censés définir un tel contexte?

Partant de l'hypothèse selon laquelle la qualité de l'environnement scolaire inclusif influence le vécu de l'adolescent en situation de handicap moteur et occasionne un retentissement psychologique chez ce dernier, les résultats de l'étude ont effectivement souligné le vécu par les adolescents, d'une certaine souffrance psychologique. Cette souffrance est marquée d'une part par l'offre de l'environnement scolaire inclusif dépourvu des infrastructures adéquates à leur situation (accès aux bâtiments, salles de classe, toilettes, infirmerie, cantine scolaire, etc.) et d'autre part, il est constaté un imbroglio dans l'offre pédagogique et les formes de médiation que le cadre d'apprentissage propose à cette catégorie d'élèves à besoins particuliers. Lenoir (2009) avait déjà évoqué la nécessité pour les intervenants à mettre en œuvre des stratégies susceptibles de faciliter l'apprentissage chez les élèves, en particulier ceux en situation de handicap.

Les résultats de l'étude mettent en relief la difficulté que rencontrent les élèves en situation de handicap au Cameroun à se faire accompagner pédagogiquement de manière à booster leur apprentissage. À travers le discours des participants, on peut remarquer que certains enseignants se démarquent tout de même par un souci manifeste de faire intervenir certains élèves en situation de handicap dans le rapport didactique. Néanmoins, il demeure la question de la formation des enseignants et des intervenants dans un contexte d'éducation inclusive au Cameroun. En effet, l'absence ou l'insuffisance dans la mise en œuvre des pratiques éducatives et de formation des intervenants auprès de cette catégorie d'élèves peut justifier le manque d'intérêt constaté dans cette étude.

Comme l'avait suggéré lonescu (1997), la qualité de vie à l'école devrait favoriser l'intégration et le bien-être des élèves à besoins spécifiques. Dans un environnement scolaire inclusif, la confrontation au handicap peut refaire surface chez le sujet concerné dans la mise à l'épreuve des problèmes d'accessibilité et des difficultés pédagogiques. Aussi, l'on peut remarquer que les dits et les non-dits liés à la situation du handicap chez le sujet, dans son rapport au corps, peuvent être exacerbés dans cet environnement scolaire inclusif; surtout si les différents acteurs n'ont pas été préparés à cette approche. Car, un établissement est dit inclusif lorsqu'il est capable de prendre en compte les différences tout en accordant un cadre contenant à tous ses apprenants. Dès lors qu'il est constaté un malaise et un mal-être chez une catégorie d'élèves notamment ceux en situation de handicap, générés par le cadre institutionnel, alors on questionne le caractère inclusif de cette institution éducative. 
II est donc important que la question de l'efficacité des établissements scolaires inclusifs au Cameroun soit pensée afin que l'école puisse prendre en compte les réalités des enfants (Teil, 2006). En outre, les différents intervenants (élèves, enseignants, administrateurs, etc.) devraient être préparés à affronter le défi de l'éducation inclusive, afin de parvenir à réduire ses effets dévastateurs sur l'élève en situation de handicap. Ceci pourrait contribuer non seulement à accroître le sentiment du vivre-ensemble, mais aussi à faciliter l'adaptation et l'apprentissage chez tous les élèves pris dans un tel contexte.

\section{RÉFÉRENCES}

Anaut, M. (2006). L'école peut-elle être facteur de résilience? Empan (3)63, 30-39.

Bacro, F. et Florin, A. (2013). Introduction. Entre complexité et richesse : la diversité des défis liés à l'intérêt des chercheurs et des professionnels pour la qualité de vie. Dans $\mathrm{F}$. Bacro (dir.), La qualité de vie-Approches psychologiques (p.7-14). Rennes, France: Presses universitaires de Rennes.

Bee, H. et Boyd, D. (2008). Les âges de la vie. Psychologie du développement humain. Québec (QC) : Édition du renouveau pédagogique.

Bon, C. (2007). Adolescence et handicap, un périlleux passage. Dans A. Ciccone., S. KorffSausse., S. Missonnier et R. Scelles (dir.), Cliniques du sujet handicapé. Actualité des pratiques et des recherches (p.95-118). Toulouse, France : ERES.

Bréjard, V. et Pedinielli, J.-L. (2014). Approche psychodynamique du handicap. Dans M. Jover (dir.), Psychologie et handicap (p. 58-72). Aix-en-Provence, France : Presses de L'Université de Provence

Cardinet, A. (1998). La médiation en France, aujourd'hui, et ses applications dans le secteur scolaire : ses références, ses significations, ses pratiques (thèse de doctorat en science de l'éducation) Lyon : Université de Lyon 2.

Cloutier, R. (1996). Psychologie de l'adolescent. Paris, France : Gaetan Morin Éditeur.

Coudronnière, C. et Mellier, D. (2016). Qualité de vie à l'école des enfants en situation de handicap. Revue de questions. Rapport commandé par le Cnesco. Récupéré de https://www.cnesco.fr/fr/qualité-de-vie-a-lecole/

Douville, O. (2006). Les méthodes cliniques en psychologie. Paris, France : Dunod.

Guimard, P., Bacro, F., Ferrière, S., Florin, A., Gaudonville, T. et Thanh-Ngo, H. (2015). Le bien-être des élèves à l'école et au collège. Validation d'une échelle multidimensionnelle, analyses descriptives et différentielles. Education et formations, 89, 163-184.

Ionescu, S. (1997). La recherche dans le domaine de la qualité de vie des personnes présentant une déficience intellectuelle. Revue francophone de la déficience intellectuelle, 1(8), 5-17.

Joly, F. (2003). Notre corps n'est rien sans le corps de l'autre. Thérapie psychomotrice (134), 40-58

Korff-Sausse, S. (1996). Le miroir brisé. L'enfant handicapé, sa famille et le psychanalyste. Paris, France : Calmann Levy

Lenoir,Y. (2009). L'intervention éducative, un construit théorique pour analyser les pratiques d'enseignement. Nouveaux cahiers de la recherche en éducation, 12(1), 9-29.

Marcelli, D. et Braconnier, A. (1988). Psychopathologie de l'adolescent. Paris, France: Masson.

MINAS (2010). Loi $\mathrm{N}^{\circ}$ 2010/002 du 13 avril 2010 portant protection et promotion des personnes handicapées au Cameroun.

Muchielli, A. (2009). Dictionnaire des méthodes qualitatives en sciences humaines et sociales ( $3^{\mathrm{e}}$ éd). Paris, France : Armand Colin.

Myara, N. (2017). Le plan d'intervention: un processus et des ententes. Montréal, QC : Éditions JFD. 
Plaisance, E. et Schneider, C. (2013). L'inclusion scolaire des enfants handicapés comme révélateur des tensions éducatives. Revue Phronesis, 2, 87-96.

Potvin, P. et Lacroix, M-E. (2009). De l'intégration à l'inclusion scolaire des élèves en difficulté d'adaptation et d'apprentissage. Récupéré de http://rire.ctreq.qc.ca/

Rask, K., Astedt-Kurki, M. et Laippala, P. (2002). Relationship among adolescent subjective well-being, health behavior and school satisfaction. Journal of School-Health, 72(6), 243249.

Tchokote, E. (2019). Autour de la déficience visuelle, des souffrances à contenir dans l'espace classe : une analyse de l'ambivalence de la fonction contenante en situation d'éducation inclusive. Dans M. Djeumeni Tchamabe., E. Voulgre., D. Groux. et S. Nyebe Atangana (dir.), Quelle école pour demain? Enjeux, priorité et défis (p. 417-422). Paris, France : Harmattan.

Teil, P. (2006). Une école qui ne prend pas en compte la réalité des enfants. Empan, 3(63) 27-29.

Terrisse, B., Kalubi, J.C., Larivée, S. (2007). Résilience et handicap chez l'enfant. Reliance, 2(24), $12-21$.

Thomazet, S. (2006). De l'intégration à l'inclusion. Une nouvelle étape dans l'ouverture de l'école aux différences. Le Français aujourd'hui, 1(152), p.19-27.

\section{RÉSUMÉ}

L'objectif de cet article est de saisir le sens de l'expression de la souffrance psychique chez l'adolescent en situation de handicap moteur inscrit dans un établissement secondaire inclusif dans un contexte d'imbroglio dans l'offre prônée par l'environnement scolaire en question. À travers la méthode clinique qualitative, l'utilisation de l'entretien clinique auprès de quatre cas d'adolescents inscrits au lycée de Biyem-Assi (Yaoundé-Cameroun), retrace l'expression de la souffrance psychique dans les discours des sujets (mentalisation par la tristesse, dépréciation de soi, angoisse, anxiété, développement d'un faux self, hyperadaptation). Sur le plan comportemental, on observe le retrait, le repli sur soi, la phobie face aux situations stressantes.

\section{MOTS CLÉS}

souffrance psychique, handicap moteur, environnement scolaire, adolescent, école inclusive

\section{ABSTRACT}

The objective is to understand the meaning of psychic suffering expressed in the adolescent with physical disability and who is enrolled in an inclusive secondary school, in an imbroglio context of the advocated offer by the school environment in question. Through the qualitative clinical method, the use of clinical interview with four adolescents cases enrolled in Biyem-Assi high school (Yaoundé- Cameroon), traces the expression of psychic suffering in the subjects' speeches including; mentalization by sadness, self-depreciation, anxiety, development of a false- self and hyper-adaptation. On the behavioral aspect, it is observed self-withdrawal and phobia in stressful situations.

\section{KEY WORDS}

psychic suffering, motor impairment, school environment, adolescent, inclusive school 